\title{
CHEMICAL HETEROGENEITY AND IMPERFECT MIXING IN THE SOLAR NEBULA ${ }^{1}$
}

\author{
DAVID J. STEVENSON \\ Division of Geological and Planetary Sciences, California Institute of Technology \\ Received 1988 June 8 ; accepted 1989 July 27
}

\begin{abstract}
It is shown that for the "standard" accretion disk model of the solar nebula, the efficiency of mixing the products of thermochemical processing from small to large disk radii depends only on the ratio of $D / v \equiv k$ ( $D=$ eddy diffusivity, $v=$ eddy viscosity). In the steady state limit, where mixing is most efficient, the fractional contamination at radius $R$ which is due to thermochemical processing at radius $R_{p}<R$ is found to be $\sim\left(R_{p} / R\right)^{\gamma}$ where $\gamma \equiv 3 / 2 k$. This assumes that accretion takes place predominantly at radii $R>R_{p}$, which is true for all but very low angular momentum models. Since most of the mass resides at large radii $R \gg R_{p}$, it is concluded that if $k \lesssim 1$, then most of the solar nebula was not contaminated by the consequences of thermochemical equilibria that were established at "small" radii (e.g., of order $1 \mathrm{AU}$ ). This condition is almost certainly satisfied if the physical process responsible for $v$ is thermal convection or waves, but has uncertain validity during the early phases of disk evolution where accretion-induced shear instabilities may dominate, as Prinn discusses. In most cases, and especially during the later most relevant stage of disk evolution, interstellar dominance is implied for most solar nebula speciation and is predicted for cometary speciation except possibly for a small contamination which is due to catalyzed hydrogenation of $\mathrm{CO}$ to $\mathrm{CH}_{4}$ and other hydrocarbons. If primordial giant planets possessed accretion disks, then the chemical speciation of the disk may have been partly that of the solar nebula. However, greater mixing and gas processing (including conversion of $\mathrm{CO}$ to $\mathrm{CH}_{4}$ and $\mathrm{N}_{2}$ to $\mathrm{NH}_{3}$ ) might have occurred in these circumstances. The formalism developed here may have applicability to the interpretation of compositional gradients in the nebulae of Young Stellar Objects, and may be relevant to the survivability of interstellar dust grains.
\end{abstract}

Subject headings: comets - planets: abundances — solar system: general — stars: accretion

\section{INTRODUCTION}

The atoms and molecules in our solar system came from the interstellar medium and suffered an uncertain amount of chemical processing before reaching their eventual resting place in the Sun, the planets, and smaller bodies. It is of fundamental importance to understand the extent to which the chemical speciation and form of this material was determined prior to arrival in the solar nebula. How much of the solid material in meteorites or comets has survived largely unaltered from the interstellar dust? Is the gas phase speciation of important atoms (oxygen, carbon, and nitrogen especially) determined prior to arrival in the solar nebula or is it determined by local processing? The answers to these questions affect how we develop our concepts of solar system and planetary origin.

Only a small amount of material is known to be clearly "exotic" (presumably presolar nebula), primarily on the basis of isotopic characteristics (Anders 1987; Wasserburg 1987). However, we should recognize that interstellar material need not necessarily have distinctive signatures (features that could not be explained by local nebula events). We should also acknowledge that our view of the solar system may be parochial, at least in respect of the solid material we currently have access to. Only a few percent (at most) of all the once solid material in the solar nebula has ended up in bodies interior to Jupiter. Certainly, chondrules and refractory inclusions are common in chrondritic meterorites and reflect heating events sufficiently severe to involve melting and at least partial evaporation and condensation. The ubiquity of these features argues

\footnotetext{
${ }^{1}$ Contribution number 4642 from the Division of Geological and Planetary Sciences, California Institute of Technology, Pasadena, California 91125.
}

for at least transient high-temperature events in the material that formed most meteorite parent bodies (see Wood 1988). We know much less about the composition and phase assemblage of comets. Interplanetary dust particles may be derived in part from comets but reflect only the involatile component of comets. Accordingly, we should approach the fundamental questions posed above with the view that our existing observational data only weakly constrain the possible answers, especially when we consider how massive the cometary reservoir may be (e.g., Spinrad 1987).

The standard picture of the solar nebula is an accretion disk: a pancake of gas and dust assembled from interstellar material. The disk is formed by gravitational collapse and has an evolution that depends on many variables: the mass infall rate, the specific angular momentum and its distribution, the opacity, and the availability of instability mechanisms to induce redistribution of angular momentum and mass and spread the disk (Lynden-Bell and Pringle 1974; Cassen, Shu, and Terebey 1985). If the specific angular momentum is small enough or the infall rate is high enough, then high temperatures $(\gtrsim 1500 \mathrm{~K})$ may be produced in much of the initially infalling material, at least for arrival locations $\$ 3 \mathrm{AU}$ from the center. Examples of calculations which create these conditions include Boss (1988). High temperatures may also be produced by local heating events such as flares (see discussion by Cameron 1988). It is important to remember, however, that the solar nebula may have been very large and had most of its mass in outer regions where accretional heating was low. A characteristic size can be estimated from the "centrifugal radius" $R_{J} \sim J^{2} / K^{2} G M^{3}$ ( $J=$ total angular momentum; $M=$ total mass; $G=$ gravitational constant; $K \simeq \frac{2}{9} ;$ see Cassen, Shu, and 
Terebey 1985). Accordingly,

$$
R_{J} \simeq(100 \mathrm{AU})\left[\frac{(J / M)}{10^{20} \mathrm{~cm}^{2} \mathrm{~s}^{-1}}\right]^{2}\left(\frac{M_{\odot}}{M}\right)
$$

If we imagine a disk with surface density $\sigma \sim \sigma_{0} X^{-\beta}$, were $X$ is the cylindrical radius in units of $1 \mathrm{AU}$ and $\sigma_{0} \sim 2 \times 10^{3} \mathrm{~g}$ $\mathrm{cm}^{-2}$, typical of estimates $\sim 1$ AU (e.g., Cassen, Shu, and Terebey 1985; Ruden and Lin 1986; Hayashi, Nakazawa, and Nakagawa 1985) then

$$
\frac{J}{M} \simeq 10^{17} \frac{X_{0}^{5 / 2-\beta}}{(5 / 2-\beta)} \mathrm{cm}^{2} \mathrm{~s}^{-1}
$$

where $X_{0}$ is the outer radius of the disk. A value of $X_{0} \sim 10^{2}$ yields $J / M \sim 10^{20} \mathrm{~cm}^{2} \mathrm{~s}^{1}$ for $\beta=1$. This value of specific angular momentum is believed appropriate for collapsing clouds. Under these circumstances, $95 \%$ of the disk mass resided beyond $5 \mathrm{AU}$. Of course, the disk is dynamic and one cannot necessarily associate specific disk localities with the formation localities of current planets for several reasons. Nevertheless, it seems reasonable to pose the issue of nebular chemical processing in the context of the following specific question: If a molecule is accreted onto the disk at cylindrical radius $R=R_{i}$ and comes finally to rest (in a planet, for example) at $R=R_{f}$, what is the probability that it visited some smaller radius $R=R_{p}$ in the intervening time? This "visit" would be a consequence of the effects of winds and diffusion. If the thermodynamic conditions at $R_{p}$ are suitable for thermochemical processing, either in the gas phase or catalyzed by grains, and if the probability of this sequence of events is high for most of the material that ends up at $R_{f}$, then we can talk of the chemical speciation in the solar nebula as a nebula-defined property. This is implicit in the work of Lewis and Prinn (1980); see an extensive review (Prinn and Fegley 1988). In their model, the gas-phase speciations of carbon and nitrogen are predominantly $\mathrm{CO}$ and $\mathrm{N}_{2}$, respectively, not necessarily because of an interstellar inheritance, but because this is the quenched speciation arising from thermochemical processing in a solar nebula of appropriate thermodynamic conditions ( $\sim 10^{-4}$ bar total pressure at $T \sim 10^{3} \mathrm{~K}$; cosmic abundances are assumed). Clearly, the probability that nebular thermochemistry is important must be greater for material accreted at small $R$ or resident at small $R$, but most of the disk and most of the condensate resides at large distances far beyond the region where gas phase thermochemistry or even some surfacecatalyzed thermochemical reactions can proceed on an interesting time scale. This paper seeks to quantify the expected trend from nebula processing to interstellar inheritance as a function of distance.

Upon first encountering this issue, it is natural to suppose that the problem is ill posed. The dynamics of the solar nebula are poorly understood, and one could imagine that a variety of assumptions could be made concerning mixing efficiency ("eddy diffusivity") in the nebula, leading to any answer one wanted. However, there is a wide range of models for which this indeterminancy does not arise. The problem is well posed in most models where the mixing process responsible for diffusing molecular species is also the process for redistributing angular momentum. This requirement is satisfied by many of the currently favored accretion disk models of the solar nebula. As explicitly demonstrated below, the efficiency of mixing (or, equivalently, the radius beyond which interstellar speciation dominates) does not depend on the magnitude of the diffusivity but only on the ratio of diffusivity to viscosity. The former is a poorly known quantity, but the latter is not expected to differ much from unity for most realizations of a turbulent medium (Canuto and Battaglia 1988; but see Prinn 1990 for a contrary view). If angular momentum is redistributed by wave motions as favored by many current workers (e.g., Larson 1989), then the diffusivity may be much less than the "viscosity"; this will strengthen the conclusions we reach in this paper (see $\S$ II). The solar nebula differs fundamentally from a planetary atmosphere or stellar interior because it is in Keplerian rotation and the distribution of material subject to this basic state can be modified by the mixing process. By contrast, a planetary atmosphere or stellar interior has a hydrostatic basic state that can persist indefinitely, irrespective of mixing, thereby allowing an arbitrary efficiency of mixing given sufficient time. The insensitivity of mixing efficiency to the magnitude of diffusivity in the solar nebula can be qualitatively understood in one of two ways. In a steady state accretion disk, doubling the diffusivity (and viscosity) also doubles the advective flow (the radially inward wind) and the mass throughput of the disk, thereby negating the faster stirring. In a nonsteady state disk, doubling the diffusivity reduces the diffusive time scale accordingly but increases the rate at which the disk evolves in a compensating way. The situation is analogous to a treadmill: no matter how fast diffusion occurs, the disk evolves faster and the diffusive effects have difficulty permeating the entire disk.

Other nebula processes can effect the chemistry, without requiring transport of molecules over large distances within the nebula. The accretion shock, lightning, photochemistry, and processing within weakly-bound (leaky) protoplanetary atmospheres are all examples of "local" processes. These are not considered in detail here and deserve more attention. The accretion shock can produce a temperature rise $\gtrsim 10^{3} \mathrm{~K}$ even out at $10 \mathrm{AU}$ for a brief period adjacent to the shock front. Lightning is intrinsically ineffective in processing large volumes of gas (Prinn and Fegley 1988), and the same authors question whether photochemistry can be significant, but this is debatable (e.g., Yung et al. 1988). Processing within protoplanetary atmospheres has been suggested as an important process (e.g., Stevenson 1985), but the efficiency of returning processed gas to the nebula is very uncertain. Protoplanets are certainly capable of processing and if they have accretion disks, they may be described by the model developed below, as we discuss.

In the next section the standard model is described and the basic equations summarized. In $\S$ III these equations are solved for the steady state case. Section IV discusses timedependent solutions, and $\S \mathrm{V}$ describes the implications of these results for the questions posed above, given specific assumptions about accretion, the ratio of diffusivity to viscosity, and the processing radius $R_{p}$.

\section{THE BASIC MODEL}

We employ a standard description of the solar nebula as an accretion disk (e.g., Cassen, Shu, and Terebey 1985; Morfill, Tscharnuter, and Völk 1985; Cameron 1988). The disk is described by a surface density $\sigma(R, t)$ which satisfies continuity

$$
\frac{\partial \sigma}{\partial t}+\frac{1}{R} \frac{\partial}{\partial R}\left(R U_{R} \sigma\right)=S(R, t),
$$

where $U_{R}$ is the radial flow and $2 \pi R S d R$ is the material accreted between $R, R+d R$ in unit time. This equation ceases to be 
correct both at very small $R$ (the protostar) and at very large $R$ (the disk periphery), where cylindrical symmetry ceases to be appropriate. If the sole cause of angular momentum redistribution within the disk is a viscous couple characterized by a kinematic viscosity $v$, then the radial velocity is prescribed

$$
U_{R}=\frac{-3}{R^{1 / 2} \sigma} \frac{\partial}{\partial R}\left(\sigma \nu R^{1 / 2}\right)+\frac{2 S(R, t) J(R, t)}{\sigma R \Omega},
$$

(e.g., Lin and Papaloizou 1985, p. 992). This assumes that the disk is locally Keplerian (angular velocity $\Omega$ ) and $J(R, t)$ is now the excess specific angular momentum of the accreting material. There is now some ebservational evidence for Keplerian motion in accretion disks of protostars (Sargent and Beckwith 1987), and theory predicts Keplerian motion because $v \ll \Omega R^{2}$. There is no well-developed theory or observational constraints on $J$, although one plausible theoretical assumption is $J \simeq$ constant at small $R$ if the collapsing cloud has constant specific angular momentum. A magnetically coupled (rigidly rotating) cloud would have $J \propto R^{2}$. Neglecting $J$, substitution of equation (4) into (3) yields a simple diffusive equation

$$
\frac{\partial \sigma}{\partial t}=\frac{3}{R} \frac{\partial}{\partial R}\left[R^{1 / 2} \frac{\partial}{\partial R}\left(\sigma \nu R^{1 / 2}\right)\right]+S .
$$

If $C(R, t)$ denotes the mole fraction of some chemical species which is a minor constituent of the $\mathrm{H}_{2}$-dominated disk, then the continuity equation including diffusion is

$$
\frac{\partial}{\partial t}(\sigma C)+\frac{1}{R} \frac{\partial}{\partial R}\left(R U_{R} \sigma C\right)=\frac{1}{R} \frac{\partial}{\partial R}\left(R \sigma D \frac{\partial C}{\partial R}\right)+Q+C_{s} S,
$$

where $D$ is the diffusivity, $C_{s}(R, t)$ is the mole fraction of the accreting material, and $Q$ represents all internal gains and losses (condensation, evaporation, transformation). From eqs. (3) and (6), we immediately find that

$$
\frac{\partial C}{\partial t}+U_{R} \frac{\partial C}{\partial R}=\frac{1}{\sigma R} \frac{\partial}{\partial R}\left(R \sigma D \frac{\partial C}{\partial R}\right)+\frac{Q}{\sigma}+\left(C_{s}-C\right) \frac{S}{\sigma} .
$$

In the calculations below, we assume

$$
D=k v
$$

where $k$ is a universal constant of order unity. In principle, $k$ may vary with position but here we avoid specific assumptions about the origin of the diffusive processes and assume that they operate in the same fashion at each $R$. If a diffusive description is valid at all, then the likely origin of $D$ and $v$ is turbulence (produced by accretion or thermal convection or wave breaking). In general, $k \neq 1$ in a turbulent system because the nonlinearities that mimic diffusion and viscosity involve different correlation functions of the small-scale velocity field. In the particular detailed analysis of convectively induced turbulence carried out by Canuto and Battaglia (1988), $k \simeq K_{0}^{3} \simeq 3$ where $K_{0} \simeq 1.5$ is the Kolmogorov constant. This result holds even after allowing for the turbulence anisotropy introduced by rotation (the Coriolis force and Keplerian shear). However, a different value of $k$ may pertain to other kinds of turbulenceinducing instabilities, as Canuto and Battaglia point out. Accordingly, we must consider $k$ to be uncertain. One could question whether a diffusive approach is applicable at all; given the current uncertainties about solar nebula dynamics, could we anticipate a process for redistribution of angular momentum or constituents that is not diffusive? The answer is that one can certainly envisage nondiffusive processes that redistribute angular momentum much more efficiently than they redistribute constituents - many wave motions have this property. It is also possible to envisage circumstances in which $v \ll D$, as Prinn (1990) points out. However, his proposal is contingent on the predominance of accretion-induced shear instabilities, which may only apply very early in the nebula evolution; and the fortuitious near-cancellation of the nonlinear effects associated with "positive" and "negative" viscosity contributions. (Of course, there need not be a true viscosity, but one can talk of an effective viscosity which mimics the angular momentum transfer.) It is difficult to assess this likelihood quantitatively, and the main conclusions reported here are accordingly conditional on $v \gtrsim D$. (In actuality, $v \gtrsim D / 5$ is probably sufficient.)

\section{STEADY STATE MODELS}

Time-independent accretion disks are implausible when viewed globally, but may approximate reality over part of the disk for a substantial fraction of their lifetime. It is instructive to examine time-independent solutions because they often have a simple fundamental form and illustrate the basic physics. From equations (3) and (7) with $\partial C / \partial t=0, Q=0$, and $\partial \sigma / \partial t=0$,

$$
\begin{aligned}
R \sigma U_{R} \frac{d C}{d R}-\frac{d}{d R}( & \left.R \sigma D \frac{d C}{d R}\right) \\
& =\left(C_{s}-C\right) R S=\left(C_{s}-C\right) \frac{d}{d R}\left(R \sigma U_{R}\right) .
\end{aligned}
$$

Define $y \equiv C_{s}-C$, so

$$
-\frac{d}{d R}\left(R \sigma U_{R} y-R \sigma D \frac{d y}{d R}\right)=0,
$$

assuming $C_{s}$ is a constant. The function $y$ can be thought of as the fractional modification of the disk composition because of processing that occurs at some small radius $R_{p}$. The assumption that $R_{p}$ is small is equivalent to the assumption that the angular momentum of the collapsing cloud is substantial, i.e., $R_{J} \gg R_{p}$, consistent with equation (1). In particular, we can represent the effects of thermodynamical processing at $R=R_{p}$ by assuming that the species in question is completely destroyed at that point. If we set $C_{s}=1$ (by convention) then $y\left(R_{p}\right)=1$. Alternatively, we can reverse the sign in the definition of $y$ and think of $y$ as the amount of material diffusing outward from $R_{p}$, if its abundance at $R_{p}$ is unity (in some arbitrary units) and there is no supply by accretion (i.e., $\left.C_{s} \equiv 0\right)$. Integrating equation (10),

$$
\frac{d y}{d R}=\frac{U_{R}}{D} y+\frac{E}{R \sigma D},
$$

where $E$ is a constant of integration. The general solution to this equation is

$$
\begin{gathered}
y=F g(R)+E g(R) \int_{R_{p}}^{R} \frac{d R^{\prime}}{R^{\prime} \sigma\left(R^{\prime}\right) D\left(R^{\prime}\right) g\left(R^{\prime}\right)} \\
g(R) \equiv \exp \left[\int_{R_{p}}^{R} \frac{U_{R}\left(R^{\prime}\right) d R^{\prime}}{D\left(R^{\prime}\right)}\right],
\end{gathered}
$$


where $F$ is a constant of integration. In those cases where the disk is unbounded (i.e., the solution to equations (3)-(7) does not cause $\sigma(R)$ to go to zero at finite $R$ ), the obvious choice is $E=0$, because $U_{R}$ is negative everywhere and $y, d y / d R \rightarrow 0$ at large $R$ :

$$
y=g(R) \text {. }
$$

This has the well-known form of a diffusive precursor, representing the upstream composition of the flowing disk by processing at $R_{p}$. (Recall that $U_{R}$ is negative.) In some cases discussed below, the disk is finite. One can then show that the correct choice is

$$
y=E^{\prime} g(R) \int_{R}^{R_{0}} \frac{d R^{\prime}}{R^{\prime} \sigma\left(R^{\prime}\right) D\left(R^{\prime}\right) g\left(R^{\prime}\right)},
$$

where $E^{\prime}$ is some constant chosen by requiring $y\left(R_{p}\right)=1$, and $R_{0}$ is the outer radius of the disk. (Strictly speaking, there cannot be a true steady state in these circumstances, but this solution can well represent the long time solution out to the peripheral boundary layer.) The above solution is dictated by the physical requirement that $y$ and $d y / d R$ are finite. In these solutions, $U_{R}$ changes sign at some finite radius within the disk, but $y(R)$ continues to monotonically decrease as $R$ increases (unlike the behavior of eq. [13]).

From equations (3) and (4)

$$
\begin{aligned}
U_{R}= & -\frac{3 A}{2 R \sigma}+\frac{1}{R \sigma} \int^{R} y S(y) d y \\
\sigma v=A & +\frac{B}{R^{1 / 2}}-\frac{1}{3 R^{1 / 2}} \int^{R} x^{-1 / 2} d x \int^{x} y S(y) d y \\
& +\frac{2 R_{p}^{1 / 2}}{3 R^{1 / 2}} \int^{R} \frac{S(y) J(y) d y}{y^{1 / 2} \Omega(y)}
\end{aligned}
$$

The integrand in eq. (12), $U_{R} / D \equiv \sigma U_{R} / k \sigma v$, is completely determined without knowing $\sigma(R), v(R)$ separately. The integration constants $A$ and $B$ are determined by the accretion rates of mass and momentum onto the proto-Sun at some small inner radius of the disk. If there is no viscous stress between the proto-Sun and the nebula, then $B=0$. Since the proto-Sun cannot accommodate angular momentum in excess of the local Keplerian value, $B / A R_{s}^{1 / 2} \lesssim 1$ where $R_{s}$ is the proto-Sun radius (inner edge of the disk).

Consider the simplifications $S=J=0$. The disk is unbounded and the solution corresponding to eq. (13) is then

$$
\begin{aligned}
y(X) & =\left[\frac{(1+\delta)}{\left(X^{1 / 2}+\delta\right)}\right]^{2 \gamma} \gamma \equiv \frac{3}{2 k}, \\
X & \equiv \frac{R}{R_{p}}, \delta \equiv \frac{B}{A R_{p}^{1 / 2}} \lesssim\left(\frac{R_{s}}{R_{p}}\right)^{1 / 2} \ll 1 .
\end{aligned}
$$

Neglecting $\delta$, we see that $y \sim X^{-\gamma}$. For the most interesting case of $y \equiv C_{s}-C$ (where $C_{s}=1$ is the "cosmic" composition by definition), we see that $C$ deviates from the cosmic value by an amount $\left(R_{p} / R\right)^{\gamma}$ at large $R$. This is a small correction, even for $k=3 \quad\left(\gamma=\frac{1}{2}\right)$. For example, if processing occurs at $T \sim 1200 \mathrm{~K}, R_{p} \sim 0.5 \mathrm{AU}$ (plausible values for the solar nebula gas phase reactions) then $C(R)$ is approaching cosmic at Jupiter $\left(R \sim 10 R_{p}\right)$.

Consider, now, the case $S(R)=S_{0} X^{-(2+\epsilon)}$. We then find (still for $J=0$ )

$$
\begin{aligned}
\sigma v & =A+\frac{B}{R^{1 / 2}}+\frac{S_{0} R_{p}^{2} X^{-\epsilon}}{3 \epsilon(1 / 2-\epsilon)}, \\
U_{R} & =\frac{-3 A}{2 R \sigma}-\frac{S_{0} R_{p}^{2} X^{-(1+\epsilon)}}{\epsilon \sigma},
\end{aligned}
$$

except for the special cases $\epsilon=0, \frac{1}{2}$ which involve logarithmic terms (but can be recovered from the above expressions with careful use of limits). There are three cases to consider: $\epsilon>\frac{1}{2}$, $0<\epsilon<\frac{1}{2}$, and $\epsilon<0$. If $\epsilon>\frac{1}{2}$, most of the accretion occurs at very small radii. This is physically unrealistic and moreover leads to solutions insignificantly different from eq. (17), so we discuss it no further. If $0<\epsilon<\frac{1}{2}$ then the accretion is still predominantly concentrated toward small $R$ but to a diminishing extent as $\epsilon$ decreases. Neglecting the correction of finite $B$ (small because $\delta \ll 1$ ), one finds

$$
\begin{gathered}
C(R) \simeq 1-\left(\frac{R_{p}}{R}\right)^{\gamma}\left\{\frac{\left[1+\left(R_{1} / R\right)^{\epsilon}\right]}{\left[1+\left(R_{1} / R_{p}\right)^{\epsilon}\right]}\right\}^{-2 \gamma \epsilon}, \\
R_{1} \equiv R_{p}\left[\frac{3 A \epsilon(1 / 2-\epsilon)}{S_{0} R_{p}^{2}}\right]^{-1 / \epsilon}
\end{gathered}
$$

For $R_{1} \gg R_{p}, R$ this is well approximated by $C(R) \simeq$ $1-\left(R_{p} / R\right)^{\gamma(1-2 \epsilon)}$. In practice, this approximation only applies for $\epsilon$ small so that $1-2 \epsilon$ is not too different from unity.

The case $\epsilon<0$ is perhaps more interesting since it corresponds to most of the accretion occurring at large distances. Formally, the solution predicts that $\sigma \rightarrow 0$ at finite $R=R_{0}$; however, the solution obtained from eq. (12) becomes unphysical near this radius because no truly steady state solution can exist near the periphery (the disk must expand). Nonetheless, the solution is well approximated by

$$
\begin{gathered}
C(R)=1-\frac{E^{\prime}}{z^{\gamma}\left(1-z^{|\epsilon|}\right)^{2 \gamma}} \int_{z}^{1} z^{\gamma-1}\left(1-z^{|\epsilon|}\right)^{2 \gamma-1} d z, \\
z=\frac{R}{R_{0}}, \quad E^{\prime} \approx \frac{\left(R_{p} / R_{0}\right)^{\gamma}}{2|\epsilon|} \\
R_{0} \equiv R_{p}\left[\frac{3 A|\epsilon|(1 / 2+|\epsilon|)}{S_{0} R_{p}^{2}}\right]^{1 /|\epsilon|}
\end{gathered}
$$

provided $R / R_{0}<1$ and not too close to unity.

In Figure 1, several illustrative examples of $y \equiv C_{s}(R)$ $-C(R)$, defined as "fractional contamination" are shown for two values of $k$ and several choices of accretion. The value of $R_{1} / R_{p}$ or $R_{0} / R_{p}$ is a measure of accretion in the sense that small (large) $R_{1}$ or $R_{0}$ implies low (high) accretion flux according as $\epsilon$ is positive (negative). However, the figure shows that the results are rather insensitive to $R_{1} / R_{p}, R_{0} / R_{p}$ or $\epsilon$, and primarily sensitive to $k=3 / 2$.

We turn finally to include the case of $J \neq 0$. If we assume constant specific angular momentum of infalling material then a reasonable choice is

$$
J=J_{0}\left[1-\left(R / R_{J}\right)^{1 / 2}\right]
$$

where $R_{J}$ is the radius at which there is no excess angular momentum, presumably a place that is a "typical" disk radius in terms of the domain of interest. For the same choice of $S$ as 




FIG. 1.-Functional form of the upstream diffusive precursor, showing the almost power-law behavior of the contamination which is due to thermodynamical processing at $R=R_{p}$. Here, "fractional contamination" represents the extent to which the thermochemical processing affects the composition at $R>R_{p}$. For example, it can represent the $\mathrm{CO} /\left(\mathrm{CH}_{4}+\mathrm{CO}\right)$ ratio in the hypothetical case that all carbon is accreted as $\mathrm{CH}_{4}$ and converted to $\mathrm{CO}$ at $R=R_{p}$. (This is an unrealistic case, as discussed in the text.) Two sets of curves are shown for two values of $k$, the ratio of diffusivity to viscosity. The different curves within each family correspond to different assumptions concerning the accretion, its functional form, and specific angular momentum: (a) $S=J=0$, (b) $J=0, S \neq 0, \epsilon=0.2, R_{1}=10^{2} R_{p},(c) J=0, S \neq 0, \epsilon=0.2, R_{0}=10^{4} R_{p},(d)$ $J$ given by eq. (24); otherwise similar to $(c),(e) J$ given by eq. (26); otherwise similar to $(c)$.

before, the solution for $U_{R}$ is unchanged (eq. [19]) but

$$
\begin{aligned}
\sigma v & =A+\frac{B}{R^{1 / 2}}+\frac{S_{0} R_{p}^{2} X^{-\epsilon}}{3 \epsilon(1 / 2-\epsilon)}\left(1-\alpha+\beta X^{1 / 2}\right), \\
\alpha & \equiv(1-2 \epsilon)\left(\frac{R_{J}}{R_{p}}\right)^{1 / 2} \\
\beta & \equiv-2 \epsilon .
\end{aligned}
$$

In general, $\alpha$ and $\beta X^{1 / 2}$ can be quite large. However, $\sigma v$ is always positive so that $S_{0}$ must be kept sufficiently small to guarantee a physically realistic solution. There is no closed form solution for $C(R)$ in this instance, but the behavior is not greatly different from previous cases, as Figure 1 illustrates for some plausible parameter choices. Alternatively, the collapsing cloud may rotate rigidly at first, because of magnetic coupling. In this case,

$$
J=J_{0}\left[\left(\frac{R}{R_{J}}\right)^{2}-\left(\frac{R}{R_{J}}\right)^{1 / 2}\right],
$$

where $R_{J}$ has the same meaning as above. This implies

$$
\begin{gathered}
\sigma v=A+\frac{B}{R^{1 / 2}}+\frac{S_{0} R_{p}^{2} X^{-\epsilon}}{3 \epsilon(1 / 2-\epsilon)} \cdot\left(1+\alpha^{\prime} X^{2}+\beta X^{1 / 2}\right), \\
\alpha^{\prime} \equiv\left(\frac{R_{p}}{R_{J}}\right)^{2} \epsilon \frac{(1-2 \epsilon)}{(2-\epsilon)}
\end{gathered}
$$

Again, there is no closed form solution and Figure 1 shows a behavior not very different than previous cases.

The important and invariant features of these results are: $(a)$ Mixing is independent of diffusivity or viscosity separately, but depends on the ratio; $(b)$ The extent of mixing decays approximately as a power law with radius beyond the radius at which the processing (destruction) occurs; $(c)$ The exponent in the power law is determined by the value of $k \equiv D / v$ and is well approximated by $3 / 2 k$ independent of the strength and form of accretion; $(d)$ The radius at which interstellar dominance $\left(C \geq \frac{1}{2}\right)$ is achieved is $\sim 2^{1 / \gamma}$ in units of $R_{p}$, the processing radius.

\section{TIME-DEPENDENT SOLÜTIONS}

Although general time-dependent solutions to equations (5) and (7) can be constructed using a Green's function approach (Lynden-Bell and Pringle 1974), the form of the results is too cumbersome to make general conclusions. It is possible to construct some examples, however, which are physically realistic and demonstrate that the general conclusions of the steady state cases are good upper bounds to the extent of mixing. We can also address the difficulty that approximately steady state solutions cease to exist near the disk periphery.

The simplest way to appreciate time-dependent behavior is by consideration of similarity solutions. Lin and Papaloizou (1985) analyze a particular postaccretion disk model, based on thermal convection as the source of turbulence, in which $v \propto \sigma^{2}$. For this choice, the similarity parameter is $\xi=$ $\left(R / R_{0}\right)\left(v_{0} t / R_{0}^{2}\right)^{-1 / 7}$ where $R_{0}$ and $v_{0}$ can usually be thought of as arbitrary radius and viscosity scales but are actually prescribed (see below). Except near the inner and outer edges of the disk, Lin and Papaloizou find $\sigma \sim t^{-5 / 14}, v \sim t^{-5 / 7}$, and $T \sim \xi^{-3 / 2} t^{-13 / 21}$. Both $\sigma$ and $v$ have only very weak dependences on $\xi$ throughout most of the disk. Upon substitution in equations (1)-(5), one finds that a similarity solution for composition $C(\xi)$ satisfies

$$
k C^{\prime \prime}(\xi)+\left[\frac{1}{7} \xi+\frac{(3 / 2+k)}{\xi}\right] C^{\prime}(\xi)=0,
$$

where it is assumed that $v_{0} / R_{0}$ is the magnitude of the inward radial advection at $\xi=1$ and $t=R_{0}^{2} / v_{0}$. A physically realistic inner boundary condition for $C(\xi)$ is $C=0$ at some fixed temperature (corresponding to the processing radius) but this will not yield a similarity solution. However, we can obtain an upper bound to the effects of diffusion on distant parts of the disk if we impose $C\left(\xi_{p}\right)=0$, where $\xi_{p}$ represents the processing radius. Accordingly, $R_{p} \sim \xi_{p} t^{1 / 7}$ moves outward (as the disk expands) and thereby increases contamination relative to a more realistic model. For $C\left(\xi_{p}\right)=0$ and $C=1$ at $\xi=\xi_{0}$ (the outer edge of the disk) we find

$$
\begin{aligned}
& C=1-\frac{1}{P} \int_{\xi}^{\xi_{0}} y^{-(1+\gamma)} \exp \left(\frac{-y^{2}}{14 k}\right) d y, \\
& P \equiv \int_{\xi_{p}}^{\xi_{0}} y^{-(1+\gamma)} \exp \left(\frac{-y^{2}}{14 k}\right) d y .
\end{aligned}
$$

This solution is identical to the steady state solutions of the previous section except for the exponential term (which introduces error function terms). The important point is that the exponential term must necessarily reduce the value of $C(\xi)$ relative to the simple nearly power law behavior of the steady-state models. Notice that equation (29) actually defines a family of solutions depending on the value of $\xi_{0}$, since the value of $\xi_{0}$ 
relative to $\sqrt{14 k}$ obviously affects the functional form of $C(\xi)$. Since $\xi_{p} \ll \xi_{0}$, the dominant behavior at long times is always the steady state power law in which the "contamination" defined as $(1-C) \propto \xi^{-\gamma}$. Clearly, the outward diffusion of the consequences of thermochemical processing never outruns the outward movement of the disk periphery, both of which scale as $t^{1 / 7}$ at long times. A different scaling of $v$ and $\sigma$ than that of Lin and Papaloizou might lead to a stronger time dependent and a stronger deviation from steady state (i.e., the coefficient 14 in eq. [29] would be smaller). This would only strengthen the main conclusions of this work.

\section{APPLICATIONS}

Gas phase thermochemical reactions are extremely strong functions of temperature because they involve activation barriers. (In the applications of interest, ions are rare and the reactions must proceed through neutral intermediates.) For this reason, it is meaningful to define a "quench" temperature above which thermodynamic equilibrium is well satisfied and below which the reaction proceeds with negligible slowness in the age of the solar system. The "processing radius" $R_{p}$ introduced earlier is the nebula position beyond which the temperature is less than quench value. Lewis and Prinn (1980) used this important concept to advocate $\mathrm{CO}$ and $\mathrm{N}_{2}$ as the dominant gas phase species of carbon and nitrogen, respectively, despite the thermodynamical preference for $\mathrm{CH}_{4}$ and $\mathrm{NH}_{3}$ throughout most of the nebula. They reasoned that the chemical reactions among gas-phase species proceed with negligible slowness except at the high temperatures $\left(T \gtrsim 10^{3} \mathrm{~K}\right)$ where $\mathrm{CO}$ and $\mathrm{N}_{2}$ are thermodynamically preferred. The nebula is then "contaminated" with the end products of this high temperature processing, by outward mixing. Unfortunately, it is very difficult to test this conjecture for several reasons. First, $\mathrm{CO}$ is also apparently the preferred gas-phase carbon species in the interstellar medium, for reasons that may be completely unrelated to the thermochemical considerations of Lewis and Prinn. For a discussion of dense cloud gas-phase carbon chemistry, see Herbst (1985). The situation with $\mathrm{N}_{2}$ is unclear, but it is quite likely that this molecule is also common in the interstellar medium. Accordingly, any evidence for $\mathrm{CO}$ and $\mathrm{N}_{2}$ dominance in the solar nebula cannot be used to support the Lewis and Prinn scenario; it may merely indicate interstellar dominance. The situation is even less clear because it is not known whether $\mathrm{CO}$ and $\mathrm{N}_{2}$ were in fact the dominant carbon and nitrogen species in the outer solar system! The best evidence is the inferred abundances in comets, where new evidence has become available because of the Halley flybys (Geiss 1987; Eberhardt et al. 1987) and to some extent from groundbased observations (A'Hearn 1988). The evidence supports CO (or species involving $\mathrm{C}-\mathrm{O}$ bonding) as the dominant volatile form of carbon. Nitrogen appears depleted, possibly because it is less readily clathrated or adsorbed on water ice than is $\mathrm{CO}$ (Lunine and Stevenson 1985; Lunine 1987). The predominance of $\mathrm{CO}$ is also supported by the high mean density of the PlutoCharon system (McKinnon and Mueller 1988).

Although a quantitative test of outer solar nebula "contamination" does not seem practical at present, future observations may clarify the situation. For this reason, an application of the results of preceding sections merits attention. Consider any feature of the interstellar pattern that is "completely" destroyed by processing at $R_{p}$. An example might be $\mathrm{CH}_{4}$ which, though not completely destroyed, may be diminished to a mixing ratio $\left[\mathrm{CH}_{4}\right] /[\mathrm{CO}] \sim 10^{-5}-10^{-7}$
(Prinn and Fegley 1988) which is effectively zero for our purposes. Based on the modeling in $\S$ III, we would predict

$$
\left.\frac{\left[\mathrm{CH}_{4}\right]}{[\mathrm{CO}]} \simeq \frac{\left[\mathrm{CH}_{4}\right]}{[\mathrm{CO}]}\right|_{\mathrm{int}}\left[1-\left(\frac{R_{p}}{R}\right)^{\gamma}\right],
$$

assuming the interstellar value exceeds $10^{-5}$. For plausible values of $R_{p} \sim 0.5 \mathrm{AU}$ (corresponding to $T \sim 1200 \mathrm{~K}$; see, for example, Ruden and Lin 1986) and $\gamma \sim 1$ (intermediate between the two values adapted in Fig. 1), we find that the deficit in $\mathrm{CH}_{4}$ which is due to thermochemical processing is only $\sim 10 \%$ at the orbit of Jupiter. (In other words, $\mathrm{CH}_{4}$ is at $90 \%$ of its accreted abundance.) Readers are invited to insert their own parameter choices, since the equations are so simple. A similar result obviously applies for any other abundance ratio, in circumstances where one is much less abundant than the other and is destoyed at $R=R_{p}$. In principle, this could also be applied to interstellar signatures in dust grains, assuming they were transported passively in the gas transport (see discussions by Morfill, Tscharnuter, and Völk 1985 and Huss 1988). Again, the quantitative calibration of the calculation is very difficult, but the qualitative aspects of the theory should be valid.

An intriguing application of the theory concerns the possibility of catalyzed reactions at relatively low temperatures. Hayatsu and Anders (1981) discuss the possible hydrogenation of $\mathrm{CO}$ to $\mathrm{CH}_{4}$ and other hydrocarbons on the surfaces of hydrated silicates; further discussion and critique can be found in Prinn and Fegley (1988). Let us suppose that $\left[\mathrm{CH}_{4}\right] /\left.[\mathrm{CO}]\right|_{\text {int }}$ is negligible but that $\mathrm{CH}_{4}$ (or a suite of hydrocarbons) is created with efficiency $\epsilon$ at $R_{p}$. The outward contamination by $\mathrm{CH}_{4}$ is then

$$
\frac{\left[\mathrm{CH}_{4}\right]}{[\mathrm{CO}]} \simeq \frac{\epsilon\left(R_{p} / R\right)^{\gamma}}{1-\epsilon\left(R_{p} / R\right)^{\gamma}} .
$$

If some comets form at $R \sim 20 R_{p} \sim 30-50 \mathrm{AU}$, then this could plausibly explain the inferred $\mathrm{CH}_{4}$ abundance of a few percent in Comet Halley (Allen et al. 1987).

Finally, let us consider the application of these ideas to the nebulae of protogiant planets. In this case, unlike the solar nebula, there is clear evidence that carbon was not in the form of CO. This follows from the ice/rock ratio of Jovian and Saturnian satellites, which is about unity, consistent with most of the oxygen being in water ice (e.g., Pollack and Bodenheimer 1989). There are three possible explanations: (1) The CO was thermodynamically converted to $\mathrm{CH}_{4}$ in the inner part of the hot, dense nebula of the giant planet; this material then contaminated the rest of the protoplanetary nebula. This is physically analogous to the solar nebula scenario of Lewis and Prinn (1980) though, of course, with a different outcome (i.e., $\mathrm{CH}_{4}$ dominance) because of the different thermodynamic conditions, as Prinn and Fegley (1989) discuss in detail. (2) The protogiant planets formed very extended, convective envelopes in which $\mathrm{CO}$ was converted to $\mathrm{CH}_{4}$. This possibility, consistent with the Prinn and Fegley view, cannot be tested by the formalism developed in this paper, since it does not involve an accretion disk. It has been advocated by Pollack and Bodenheimer (1989) on the basis of their analysis of giant planet formation. However, it depends on the assumed opacity of the accreting material which is uncertain by several orders of magnitude since the dust component may be largely eliminated by coagulation at this stage. (3) The gas accreted onto Jupiter and Saturn did not have carbon mainly in the form of $\mathrm{CO}$ but 
rather as $\mathrm{CH}_{4}$ and heavier hydrocarbons. This is marginally consistent with hydrogenation as discussed above (eq. [31]).

We consider here the first of the three possibilities: Can an accretion disk efficiently convert incoming $\mathrm{CO}$ into $\mathrm{CH}_{4}$ and redistribute the $\mathrm{CH}_{4}$ so that the formation region of Callisto (say) was $\mathrm{H}_{2} \mathrm{O}$ rich and $\mathrm{CO}$ poor? This is a more complicated issue than the solar nebula calculations presented earlier because $\mathrm{H}_{2} \mathrm{O}$ can be created from $\mathrm{CO}$ and removed by condensation. Consider the following calculation: $\mathrm{CO}$ is added to the disk, transported into $R_{p}$ where the oxygen is converted to $\mathrm{H}_{2} \mathrm{O}$, which then diffuses out to $R_{s} \sim 5 R_{p}$ where the water condenses. In this case, the steady state problem for the thermodynamically produced water involves a source at $R_{p}$ and a sink at $R_{s}$. The solution for the mixing ratio of this water, denoted by $C$, is accordingly (eq. [12])

$$
\begin{gathered}
C(R)=g(R) \cdot\left[\int_{R}^{R_{s}} f\left(R^{\prime}\right) d R^{\prime} / \int_{R_{p}}^{R_{s}} f\left(R^{\prime}\right) d R^{\prime}\right], \\
f(R) \equiv[R \sigma(R) D(R) g(R)]^{-1}
\end{gathered}
$$

where $C\left(R_{p}\right) \equiv 1$ is the choice of normalization and $C\left(R_{s}\right)=0$ because of condensation. The outward flux of water at $R=R_{s}$ is accordingly

$$
F_{\mathrm{H}_{2} \mathrm{O}}=-2 \pi\left[R \sigma D \frac{d C}{d R}\right]_{R=R_{s}}[\mathrm{CO}]
$$

where $[\mathrm{CO}]$ is the $\mathrm{CO}$ mixing ratio of the accreting gas. From eq. (32), this simplifies to

$$
\begin{aligned}
F_{\mathrm{H}_{2} \mathrm{O}} & =\frac{-2 \pi[\mathrm{CO}]}{\int_{R_{p}}^{R_{s}} f\left(R^{\prime}\right) d R^{\prime}}, \\
& \simeq 2 \pi \alpha \sigma\left(R_{s}\right) D\left(R_{s}\right)[\mathrm{CO}]\left(\frac{R_{p}}{R_{s}}\right)^{\gamma},
\end{aligned}
$$

where $\alpha$ is some constant that depends on the functional forms of $\sigma D$ and the value of $k$. Typically, $\alpha \sim 1$ and the flux of $\mathrm{H}_{2} \mathrm{O}$ into the zone of condensation is less than the incoming flux of $\mathrm{CO}$ by a factor of several. This is still a large amount of water (see a similar calculation for the solar nebula; Stevenson and Lunine 1988) although clearly not enough to recreate the observed ice/water ratio for both Ganymede and Callisto if all the accreted carbon were in the form of CO. These calculations suggest that of the three possibilities listed above, an efficiently mixed accretion disk is not the sole explanation for the ice/rock ratio of satellites. This is clearly a more difficult application of the ideas of this paper, however, because of our current poor understanding of accretion conditions for giant planets.

\section{DISCUSSION}

The solar nebula was large, probably over 2 orders of magnitude larger than the linear dimension of the region within which thermochemistry could occur on the relevant time scale ( $\lesssim 10^{6}$ year). It is not surprising, therefore, that interstellar speciation might dominate most of the disk. The work reported here quantifies this possibility and shows that the extent of interstellar dominance does not depend on the magnitude of the diffusivity ( very uncertain quantity), but only on the ratio of this parameter to the viscosity, the parameter which determines the evolution of the mass distribution. This ratio $k$ is probably of order unity or less except in the special case discussed by Prinn (1990). The conclusion of interstellar dominance is especially strong if angular momentum transfer is largely by waves, as many workers now favor (Larson 1989). The radius beyond which interstellar speciation dominates is found to be $\sim R_{p} 2^{1 / \gamma}$ where $\gamma=3 / 2 k$ and $R_{p}$ is the radius within which the thermochemical speciation prevails. Unfortunately, the value of $k$ is not well enough known to enable precise predictions. Future astronomical observations (perhaps millimeter interferometry) may identify compositional gradients around Young Stellar Objects from which it would be possible to calibrate the model.

The biggest deficiency of the modeling presented here is that it focuses on only one aspect of chemical processing. Clearly, even a short period spent at high temperatures can obliterate the consequences of long periods spent at low temperatures. Infrequent, active bursts of activity (FU Orionis events, spiral density waves) might dominate the chemical processing despite their low duty cycle. However, the influence of these processes is still likely to be concentrated in the inner part of the disk, or to influence the material that accretes earliest (and ends up primarily in the Sun). Further numerical modeling is needed to clarify the role of these more complicated considerations.

This work began while the author was on sabbatical at the Department of Astronomy, Cornell University. Their support is gratefully acknowledged. Comments from a reviewer (S. Weidenschilling), and spirited criticism from R. Prinn helped clarify the issues presented. This work is supported by NASA grant NAGW-185.

\section{REFERENCES}

A'Hearn, M. F. 1988, Ann. Rev. Astr. Ap., 16, 273.

Allen, M., Delitsky, M., Huntress, W. T., Yung, Y., Ip, W.-I. Schwenn, R., Balsiger, H., and Geiss, J. 1987, Astr. Ap., 187, 502.

Anders, E. 1987, Phil. Trans. Roy. Soc. London, A, 323, 287.

Boss, A. P. 1988, Science, 241, 565.

Cameron, A. G. W. 1988, Ann. Rev. Astr. Ap., 26, 441.

Canuto, V. M., and Battaglia, A. 1988, Astr. Ap., 193, 313.

Cassen, P., Shu, F. H., and Terebey, S. 1985, in Protostars and Planets II, ed. D. C. Black and M. S. Matthews (Tucson: University of Arizona Press, p. 448.

Eberhardt, P. 1987, Astr. Ap., 187, 481.

Geiss, J. 1987, Astr. Ap., 187, 859

Hayashi, C., Nakazawa, K., and Nakagawa, Y. 1985, in Protostars and Planets II, ed. D. C. Black and M. S. Matthews (Tucson: University of Arizona Press), p. 1100 .

Hayatsu, R., and Anders, E. 1981, Topics in Current Chemistry (Berlin: Springer), $99,1$.

Herbst, E. 1985, in Protostars and Planets II, ed. D. C. Black and M. S.

Matthews (Tucson: University of Arizona Press), p. 668

Huss, G. R. 1988, Moon and Planets, 40, 165.
Larson, R. B. 1989, in The Formation and Evolution of Pिlanetary S̈ystems, ed. H. A. Weaver and L. Danly (Cambridge: Cambridge University Press), p. 31. Lewis, J. S., and Prinn, R. 1980, Ap. J., 238, 357.

Lin, D. N. C., and Papaloizou, J. 1985, in Protostars and Planets II, ed. D. C. Black and M. S. Matthews (Tucson: University of Arizona Press), p. 981.

Lunine, J. I. 1987, Bull. AAS, 19, 887.

Lunine, J. I., and Stevenson, D. J. 1985, Ap. J. Suppl., 58, 493.

Lynden-Bell, D., and Pringle, J. E. 1974, M.N.R.A.S., 168, 603.

McKinnon, W. B., and Mueller, S. 1988, Nature, 335, 240.

Morfill, G. E., Tscharnuter, W., and Völk, H. J. 1985, in Protostars and Planets II, ed. D. C. Black and M. S. Matthews (Tucson: University of Arizona Press), p. 498.

Pollack, J. B., and Bodenheimer, P. 1989, in Origin and Evolution of Planetary and Satellite Atmospheres, ed. J. Pollack and M. Matthews (Tucson: University of Arizona Press), p. 564.

Prinn, R. G. 1990, Ap. J., 348, 725

Prinn, R. G., and Fegley, B. 1989, in Origin and Evolution of Planetary and Satellite Atmospheres, ed. J. Pollack and M. Matthews (Tucson: University of Arizona Press), p. 78.

Ruden, S. P., and Lin, D. N. C. 1986, Ap. J., 308, 883. 
Sargent, A. I., and Beckwith, S. 1987, Ap. J., 323, 294

Spinrad, H. 1987, Ann. Rev. Astr. Ap., 25, 231.

Stevenson, D. J. 1985, Icarus, $62,4$.

Stevenson, D. J., and Lunine, J. I. 1988, Icarus, 75, 146

Iol Wasserberg, G. J. 1987, Earth Planet. Sci. Letters, 86, 129.

Wood, J. A. 1984, Earth Planet Sci. Letters., 70, 11.

.1988, Ann. Rev. Earth Planet. Sci., 16, 53

Yung, Y. L., Friedl, R. R., Pinto, J. P., Bayes, K. D., and Wen, J-S. 1988, Icarus, 74, 121.

D. J. Stevenson: Division of Geological and Planetary Sciences, California Institute of Technology, Pasadena, CA 91125 